This is a peer-reviewed, accepted author manuscript of the following article: Wang, X., Chen, L., Zheng, L., Fu, Z., Cooper, A. I., Zhao, C., Wang, X., Chong, S. Y., McBride, F., Raval, R., Bilton, M., Liu, L., Sprick, R. S., \& Wu, X. (2020). Covalent organic framework nanosheets embedding single cobalt sites for photocatalytic reduction of carbon dioxide. Chemistry of Materials, 32(21), 9107-9114.

https://doi.org/10.1021/acs.chemmater.0c01642

\title{
Covalent organic framework nanosheets embedding single cobalt sites for photocatalytic reduction of carbon dioxide
}

\author{
Xiaoyan Wang, ${ }^{1}$ Zhiwei Fu, ${ }^{1}$ Lirong Zheng, ${ }^{2}$ Chengxi Zhao,, ${ }^{1,3}$ Xue Wang, ${ }^{1,4}$ Samantha Y. Chong, ${ }^{1}$ Fio- \\ na McBride, ${ }^{5}$ Rasmita Raval, ${ }^{5}$ Matthew Bilton, ${ }^{6}$ Lunjie Liu, ${ }^{1}$ Xiaofeng Wu, ${ }^{1}$ Linjiang Chen, ${ }^{1,4^{*}}$ Reiner \\ Sebastian Sprick, ${ }^{1,7^{*}}$ and Andrew I. Cooper ${ }^{1,4^{*}}$
${ }^{1}$ Department of Chemistry and Materials Innovation Factory, University of Liverpool, 51 Oxford Street, Liverpool L7 3NY, UK.
${ }^{2}$ Beijing Synchrotron Radiation Facility, Institute of High Energy Physics, Chinese Academy of Sciences, Beijing 100049, P. R. China

${ }^{3}$ Key Laboratory for Advanced Materials and School of Chemistry and Molecular Engineering, East China University of Science and Technology, Shanghai, P. R. China

${ }^{4}$ Leverhulme Research Centre for Functional Materials Design, University of Liverpool, 51 Oxford Street, Liverpool L7 $3 N Y$, UK.

${ }^{5}$ Open Innovation Hub for Antimicrobial Surfaces, Surface Science Research Centre, Department of Chemistry, University of Liverpool, Oxford Road, Liverpool L69 3BX, UK.

${ }^{6}$ Imaging Centre at Liverpool, University of Liverpool, 1-3 Brownlow Street, Liverpool L69 3GL, UK.

7 Department of Pure and Applied Chemistry, University of Strathclyde, Thomas Graham Building, 295 Cathedral

Street, Glasgow G1 1XL, UK.

\begin{abstract}
Covalent organic framework nanosheets (CONs), fabricated from two dimensional covalent organic frameworks (COFs), present a promising strategy for incorporating atomically distributed catalytic metal centers into welldefined pore structures with desirable chemical environments. Here, a series of CONs was synthesized embedding single cobalt sites that were then evaluated for photocatalytic carbon dioxide reduction. A partially fluorinated, cobalt-loaded CON produced $10.1 \mu \mathrm{mol}$ carbon monoxide with a selectivity of $76 \%$, over 6 hours irradiation under visible light (turnover number $=28.1$ ) and a high external quantum efficiency of $6.6 \%$ under $420 \mathrm{~nm}$ irradiation in the presence of an iridium dye. The CONs appear to act as a semiconducting support, facilitating charge-carrier transfer between the dye and the cobalt centers, and this results in a performance comparable with the state-of-the-art heterogeneous catalysts in the literature under similar conditions. The ultra-thin CONs outperformed their bulk counterparts in all cases, suggesting a general strategy to enhance the photocatalytic activities of COF materials.
\end{abstract}

\section{INTRODUCTION}

The conversion of carbon dioxide into chemical fuels is thought to be one potential avenue to address the rising levels of $\mathrm{CO}_{2}$ in the atmosphere and the rising energy demand of our growing population. ${ }^{1-3}$ Photocatalytic reduction of $\mathrm{CO}_{2}$ has been extensively studied using homogeneous ${ }^{4,5}$ and heterogeneous catalysts. 6-8 Homogeneous catalysts usually possess high initial photocatalytic activity and selectivity for the de- sired products but they are often not stable during extended operation. The use of expensive precious metals, coupled with the difficulty in catalyst recovery and product separation, makes such homogeneous catalysis expensive. ${ }^{9,10}$ Heterogeneous catalysts can address some of these issues because they can be isolated by simple filtration and can show good stability, but are often hampered by their relatively low catalytic activity. One strategy to achieve the combined advantages of homo- and heterogeneous catalysis is to anchor individual 
catalytic metal centers on supports, yielding the so-called single-atom or single-site catalysts. It is, however, a significant challenge to construct robust single-atom catalysts, which requires the mononuclear metal complexes or single metal atoms to be atomically distributed and strongly bound to the support, ideally also incorporating functional synergy between the catalytic sites and the support. Recently, metalorganic frameworks (MOFs) ${ }^{11-13}$ and covalent organic frameworks (COFs) ${ }^{14-16}$ have been reported incorporating singleatom catalysts. Their well-defined pore structures can be tuned to provide optimal confinement of known, catalytically
active
coordination
metal
complexes. ${ }^{17,1}$

Figure 1. (a) Synthesis of Py-COF and FPy-COF, and fabrication of Co-Py-CON and Co-FPy-CON. (b, c) Experimental diffraction pattern (red), profile calculated from Le Bail fitting (black) and residual (blue), and pattern simulated from the structural model (green) for Py-COF (b) and FPy-COF (c); reflection positions are shown by tick marks. The observed peak shift can be accounted for 
by a small difference in the experimental and simulated unit cells. (d) Nitrogen adsorption isotherms (filled symbols) and desorption isotherms (open symbols) for Py-COF, FPy-COF, Co-Py-CON and Co-FPy-CON, recorded at 77.3 K.

Alternatively, molecular catalysts can be chemically tethered together in the form of a COF or MOF to afford heterogeneous catalysts, possessing spatially arranged catalytic sites and a precise control of the chemical environment around them. ${ }^{14,19,20}$ MOFs have been shown to be active for photocatalytic for $\mathrm{CO}_{2}$ reduction, producing $\mathrm{CO}$ or formate.21-24 Similarly, two-dimensional (2D) COFs have been reported as highly active photocatalysts for hydrogen production ${ }^{25-27}$ and $\mathrm{CO}_{2}$ reduction, $14,28,29$ and they have the potential to be ideal supports for anchoring atomically distributed metal centers. Comparing COFs to MOFs, the former are composed of covalent bonds rather than coordination bonds, often making (though not always) making them more stable. COFs can be conjugated in two dimensions, unlike MOFs which are usually not, offering a faster response to photoexcitation. ${ }^{14}$ Moreover COFs can be exfoliated, further increasing the surface area that can be in contact with the reaction medium. Also, COFs often have narrow band-gaps allowing efficient utilization of the solar spectrum.

Examples of COFs include materials that contain bipyridinebased units which have been previously used to stabilize metal atoms within the framework. ${ }^{14,15}$ Although these 2D COF

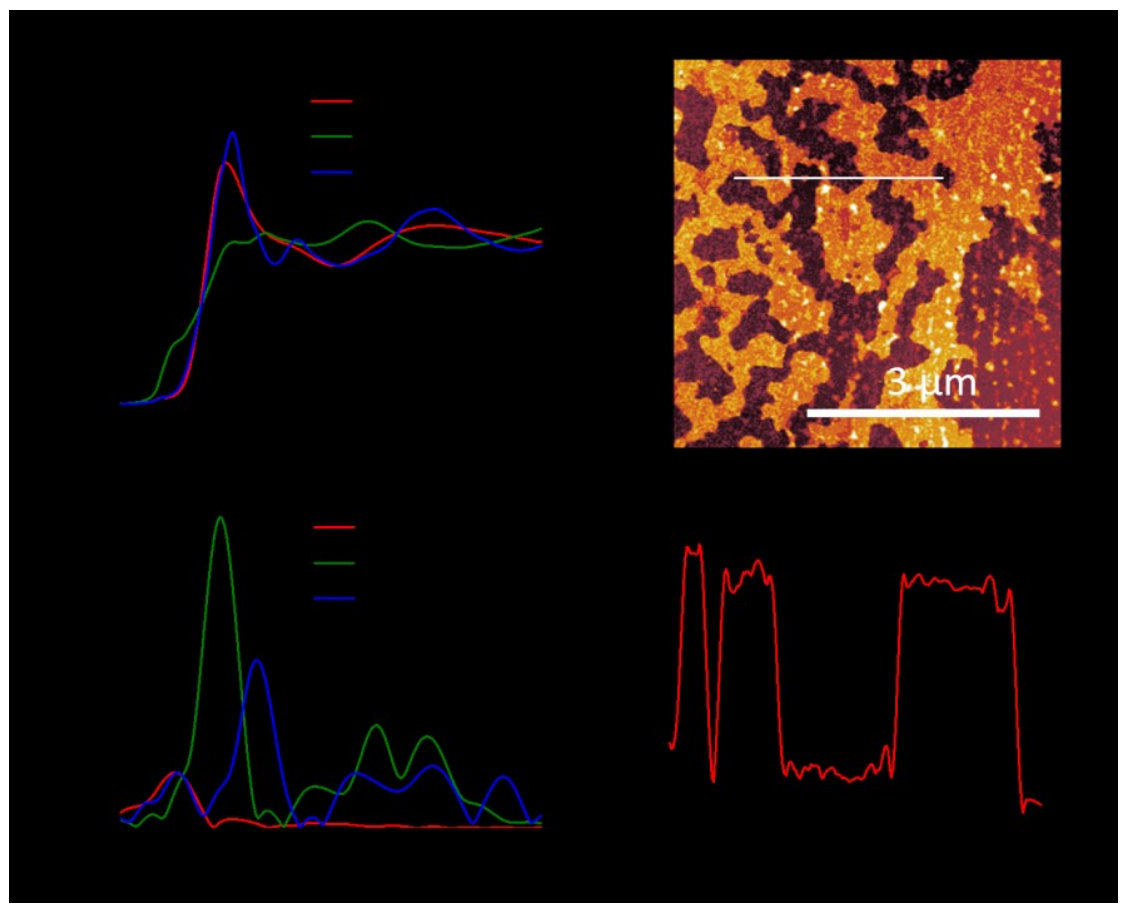

Figure 2. (a, b) XANES spectra at the Co K-edge (a), and $\mathrm{k}^{3}$-weighted Fourier-transformed Co K-edge EXAFS spectra (b) of Co-FPy$\mathrm{CON}, \mathrm{CoO}$ and $\mathrm{Co}$ foil. (c) AFM image of Co-FPy-CON, with measured thickness along the line shown in (d).

materials show strong visible light absorption, utilization of the catalytic metal sites has often been shown to be poor, as expressed by their low turnover numbers (TONs). ${ }^{30}$

Here, we report a new 2D COF, Py-COF, synthesized via a Schiff base condensation reaction, that bears metal coordination sites-iminopyridine moieties - that are formed by pyridine units and their adjacent imine groups. A partially fluorinated analogue of Py-COF, FPy-COF, was synthesized with the aim of improving the COF's binding affinity toward $\mathrm{CO}_{2}$, thus enhancing its ability to enrich $\mathrm{CO}_{2}$ around the catalytic sites. Exfoliation of bulk 2D COFs yielded covalent organic nanosheets (CONs), which were then loaded with cobalt for photocatalytic $\mathrm{CO}_{2}$ reduction. We also show that the iminopyridine moiety is a versatile metal coordination site for different transition metals, which has not yet been explored in the context of COF catalysts.

\section{EXPERIMENTAL SECTION}

COF synthesis. All COFs were prepared using a procedure based on the method described here for the synthesis of FPyCOF. A Schlenk tube was charged 5,5',5',5"'-(pyrene-1,3,6,8tetrayl)tetrapicolinaldehyde $(24.9 \mathrm{mg}, 0.04 \mathrm{mmol}), 4,4^{\prime}-$ 
diamino-2,2'-difluorobiphenyl (17.6 $\mathrm{mg}, 0.08 \mathrm{mmol}), \quad 1,2-$ dichlorobenzene $(0.5 \mathrm{~mL}), n$-butanol $(0.5 \mathrm{~mL})$, and aqueous acetic acid $(0.1 \mathrm{~mL}, 6 \mathrm{M})$. This mixture was homogenized by ultrasonication for 10 minutes and the tube was then flashfrozen at $77 \mathrm{~K}$ (liquid $\mathrm{N}_{2}$ bath) and degassed by three freezepump-thaw cycles. The tube was then sealed off and then heated at $120{ }^{\circ} \mathrm{C}$ for 7 days. The yellow precipitate was collected by centrifugation (3000 $\mathrm{min}^{-1}, 3$ minutes) and washed with $N, N$-dimethylformamide $(100 \mathrm{~mL})$, THF $(100 \mathrm{~mL})$ and anhydrous acetone $(200 \mathrm{~mL})$. After drying at $120{ }^{\circ} \mathrm{C}$, the product was obtained a yellow powder $(35 \mathrm{mg}, 88 \%)$. Anal. Calcd for $\left(\mathrm{C}_{68} \mathrm{H}_{50} \mathrm{~F}_{4} \mathrm{~N}_{8}\right)_{n}$ : C, 77.40; H, 4.78; F, 7.2; N, 10.62 . Found: C, 70.68; H, 3.48; N, 9.06.

Cobalt loading onto CONs. All cobalt loading procedures were based on the method described here for the synthesis of Co-FPy-CON. FPy-COF (20 mg) was mixed with $\mathrm{CoCl}_{2} \cdot 6 \mathrm{H}_{2} \mathrm{O}$ ( $9 \mathrm{mg}, 0.0378 \mathrm{mmol})$ in acetonitrile $(20 \mathrm{~mL})$ and the resulting suspension was ultrasonicated for 6 hours at room temperature. After this the solid was filtered off and washed with of acetonitrile $(200 \mathrm{~mL})$. The resulting solid was dried under vacuum at $60{ }^{\circ} \mathrm{C}$ overnight to give Co-FPy-CON. The cobalt content of Co-FPy-CON was determined by inductively coupled plasma-optical emission spectrometry to be $2.1 \mathrm{wt}$. \%.

Carbon dioxide reduction experiments. A quartz flask was charged with the COF nanosheets powder (1 mg), 2,2'bipyridyl $(1.5 \mathrm{mg})$, acetonitrile, water and triethanolamine (3:1:1 vol. mixture, $5 \mathrm{~mL}$ ) and sealed with a septum. The resulting suspension was ultrasonicated for 5 minutes and then purged with $\mathrm{CO}_{2}$ for 5 minutes. The reaction mixture was illuminated with a $300 \mathrm{~W}$ Newport Xe light source (Model: 6258 , Ozone free) equipped with a $\lambda>420 \mathrm{~nm}$ cut-off filter. Gaseous products were taken with a gas-tight syringe and run on a Shimadzu GC-2014 gas chromatograph equipped with a ShinCarbon ST micropacked column (Restek 80-100 mesh, $2 \mathrm{~m}$ length, $0.53 \mathrm{~mm}$ inner diameter) and a thermal conductivity detector calibrated against standard gas mixtures of known concentration.

\section{RESULTS AND DISCUSSION}

Two COFs, Py-COF and FPy-COF, were synthesized via a Schiff base condensation of 5,5',5",5"'-(pyrene-1,3,6,8tetrayl)tetrapicolinaldehyde with aromatic diamines (Figure 1a). The COFs were then ultrasonicated in acetonitrile containing $\mathrm{CoCl}_{2}$, resulting in exfoliated, Co-loaded $\mathrm{COF}$ nanosheets, Co-Py-CON and Co-FPy-CON (Figure 1a). Fouriertransform infrared spectroscopy (FT-IR) spectra of the materials were similar before and after ultrasonication in the presence of $\mathrm{CoCl}_{2}$ (Figure S2), indicating that the COFs did not undergo decomposition during delamination and metal loading. Experimental powder X-ray diffraction (PXRD) patterns of Py-COF show diffraction peaks at $3.27,4.77,6.55,7.56$, and $9.83^{\circ}$, assigned to the (110), (210), (220), (130) and (330) planes, respectively (Figure $1 \mathrm{~b}$ ). The isostructural FPy-COF shows similar levels of crystallinity and the diffraction peaks at $3.20,6.50,7.30$ and $9.62^{\circ}$ were assigned to the (110), (220), (130) and (330) planes, respectively (Figure 1c). Unit cell parameters extracted by Le Bail refinements are similar to the parameters obtained by structure simulations. The simulated diffraction profile for the AA stacking model for both Py-COF (Figure 1b) and FPy-COF (Figure 1c) agree well with the experimental PXRD data. Both Co-Py-CON and Co-FPy-CON are less crystalline than their pristine COF counterparts (Figures
S7 and S8), as a result of the processes of exfoliation and metal loading. ${ }^{31}$

Nitrogen sorption experiments were performed at $77 \mathrm{~K}$ and the Brunauer-Emmett-Teller (BET) surface areas of Py-COF and FPy-COF were found to be 924 and $1136 \mathrm{~m}^{2} \mathrm{~g}^{-1}$, respectively. These experimental surface areas are lower than those derived from atomistic models of perfectly crystalline structures (2168 and $2044 \mathrm{~m}^{2} \mathrm{~g}^{-1}$, respectively). ${ }^{25,28}$ The pore diameters derived for Py-COF and FPy-COF by fitting nonlocal density functional theory models to the $\mathrm{N}_{2}$ isotherms were 24.5 and $23.8 \AA$, respectively. Both COFs gave rise to nitrogen isotherms with shapes consistent with mesoporosity and sequential, multilayer pore filling (Figure 1d). Exfoliation and metalation resulted in a reduction of the BET surface area to 207 and $238 \mathrm{~m}^{2} \mathrm{~g}^{-1}$ for Co-Py-CON and Co-FPy-CON, respectively, though both materials were still microporous. Co-FPy$\mathrm{CON}$ shows higher $\mathrm{CO}_{2}$ adsorption amounts and larger isosteric heats than its non-fluorinated counterpart Co-Py-CON. Likewise, the bulk FPy-COF material shows a better $\mathrm{CO}_{2}$ adsorption performance than its non-fluorinated counterpart (Figures S9-S12). This can be attributed to the enhanced binding between $\mathrm{CO}_{2}$ and the fluorinated moieties of the COF, owing to the latters ability to polarize $\mathrm{CO}_{2} \cdot{ }^{32,33}$

The cobalt species in Co-FPy-CON were studied using X-ray absorption spectroscopy (XAS) at the Co K-edge, in reference to standard Co foil and cobalt (II) oxide (CoO). The Co K-edge absorption edge position for Co-FPy-CON is similar to CoO, meaning that the Co atoms on Co-FPy-CON are in the oxidation state of +2 . These results are consistent with the X-ray photoelectron spectroscopy (XPS) data (Figure S19). The coordination environment of cobalt in Co-FPy-CON was further investigated by extended X-ray absorption fine structure spectroscopy (EXAFS). Fourier transformed R-space spectrum of Co foil shows a sharp peak at around 2.2 A. (Figure $2 \mathrm{~b}$ ), corresponding to the Co-Co bond, which is absent in the spectrum of Co-FPy-CON. Similarly, the peak centered at $2.6 \AA$ that corresponds to the shortest $\mathrm{Co}$-.C Co distance in $\mathrm{CoO}$ is also absent for Co-FPy-CON. For Co-FPy-CON, the only distinct peak is centered around $1.6 \AA$, which can be attributed to the Co-N bonds, ${ }^{34}$ in line with the first peak (at $1.6 \AA$ ) of the CoO spectrum being assigned to the Co-O bond. By EXAFS curve fitting (Figure S20 and Table S3), the coordination number of the isolated Co atoms in Co-FPy-CON was determined to be 6.0, with a bond length of $2.13 \AA$. This shows that the Co atom is ligated onto the iminopyridine moiety of Co-FPy-CON, with the solvent acetonitrile molecules also participating the coordination with Co, as shown in the schematic diagram in Figure 1a. Although there are three different types of Co-N bonds in the Co-FPy-CON, only one peak in the EXAFS spectrum is assigned to the Co-N pair. This is because of the small differences in the Co-N bond lengths, approximately $0.1 \AA, 35,36$ which are not distinguishable in EXAFS spectra. These EXAFS results show that short Co..Co distances are not present in Co-FPy-CON, hence Co centers can be considered atomically distributed in the material. In final catalytic system, the additional 2,2'-bipyridine was required to form catalytic Co centers (Figure 1a). ${ }^{14}$ 


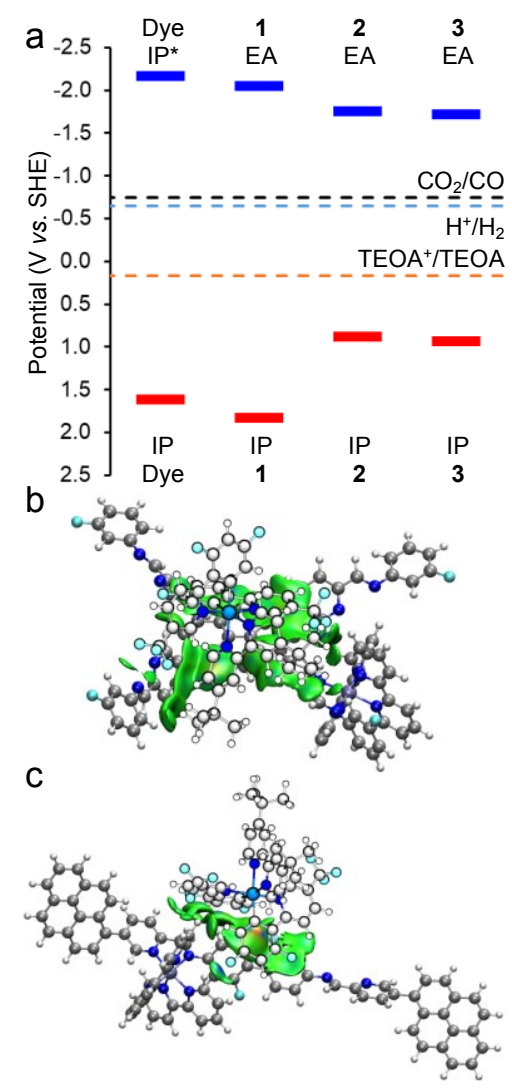

Figure 3. (a) (TD-)DFT predicted potentials of the free charge carriers (IP and EA) and excitons (IP*) of the dye, the molecular catalyst $\left[\mathrm{Co}(\mathrm{Bpy})_{3}\right]^{2+}(\mathbf{1})$, and representative fragments $[\mathrm{Co}-\mathrm{Py}(\mathrm{L})]^{2+}(2)$ and $[\mathrm{Co}-\mathrm{FPy}(\mathrm{L})]^{2+}(3)$ of Co-Py-COF and CoFPy-COF, respectively. Dashed colored lines indicate the potentials for $\mathrm{CO}_{2}$ reduction to $\mathrm{CO}$, proton reduction, and triethanolamine (TEOA) oxidation, respectively. DFT-optimized adsorption configuration of the dye on the pyrene moiety (b) or the difluorobiphenyl moiety (c) of Co-FPy-COF. Colored isosurfaces are intermolecular interactions identified and quantified by non-covalent interaction analyses. COF fragments are shown in full atomic, ball-and-stick representation, with the dye shown as a lighter sketch.

Scanning electron microscopy images (SEM) for both Co-PyCON and Co-FPy-CON show a microball-like agglomerate morphology (Figure S13). Elemental mapping images of highangle annular dark-field scanning transmission electron microscopy (HAADF-STEM) for Co-Py-CON (Figure S14) show uniform distribution of $\mathrm{C}, \mathrm{N}, \mathrm{Cl}$ and $\mathrm{Co}$ in the material. HAADFSTEM for Co-FPy-CON show that C, N, F, Cl and Co are distributed uniformly over the sample (Figure S15). Co clusters or nanoparticles were not observed by HAADF-STEM (Figure S17a), indicating that the Co species were too small to be resolved. Aberration-corrected HAADF-STEM was therefore used to characterize the Co-FPy-CON. Figure S17b shows that 


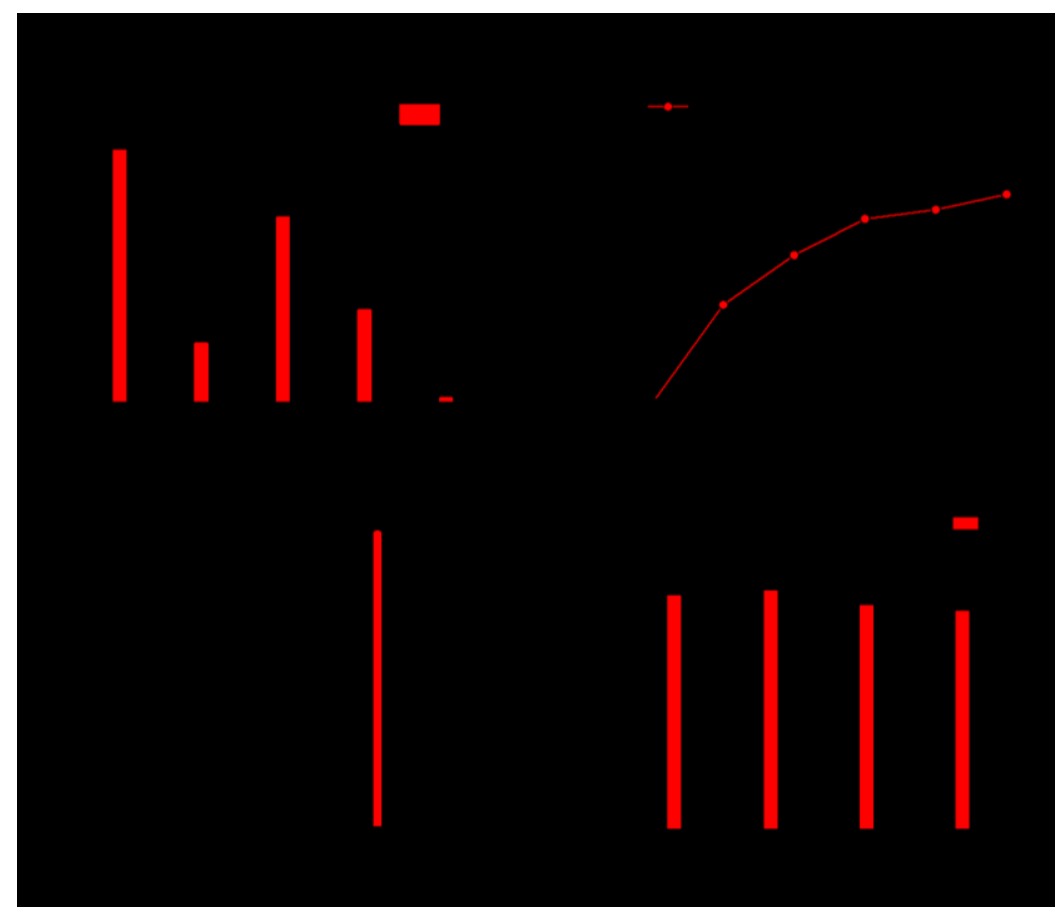

Figure 4. (a) $\mathrm{CO}$ and $\mathrm{H}_{2}$ production by the nanosheet (denoted CON) and bulk (denoted COF) of Co-FPy-COF, Co-Py-COF and CoBp-COF, over 6 hours under visible-light irradiation $\left(\lambda>420 \mathrm{~nm}, 300 \mathrm{~W}\right.$ Xe light source) with $\left(\operatorname{Ir}\left[\mathrm{dF}\left(\mathrm{CF}_{3}\right) \mathrm{ppy}\right]_{2}(\mathrm{dtbpy}) \mathrm{PF}_{6}\right.$ as photosensitizer. (b) TONs of CO production by Co-FPy-CON and [Co(Bpy) $]^{2+}$ under visible-light irradiation $(\lambda>420 \mathrm{~nm}, 300 \mathrm{~W}$ Xe light source) with $\left(\operatorname{Ir}\left[\mathrm{dF}\left(\mathrm{CF}_{3}\right) \text { ppy }\right]_{2}(\mathrm{dtbpy}) \mathrm{PF}_{6}\right.$ as photosensitizer. (c) Mass spectrum of ${ }^{13} \mathrm{CO}$ produced using Co-FPy-CON as the catalyst in the photocatalytic reduction of ${ }^{13} \mathrm{CO}_{2}$; inset: the corresponding gas chromatogram. (d) $\mathrm{CO}$ and $\mathrm{H}_{2}$ production by Co-FPy-CON over multiple 2-hour repeat runs. The sample was degassed and $1 \mu \mathrm{mol}$ of ( $\left.\operatorname{Ir}\left[\mathrm{dF}\left(\mathrm{CF}_{3}\right) \mathrm{ppy}\right]_{2}(\mathrm{dtbpy})\right)_{\mathrm{PF}} \mathrm{w}_{\mathrm{w}}$ added after each run. isolated Co atoms are distributed on the COF matrix, identified by the bright dots with some highlighted by the red cycles. Atomic force microscopy (AFM) images of Co-FPy-CON show that the nanosheets were thin stacks of the COF layers with thicknesses ranging from 1.9-4.4 nm (Figure 2c,d).

Next we used density functional theory (DFT) and timedependent (TD-)DFT calculations-performed on representative molecular models $[\mathrm{Co}-\mathrm{Py}(\mathrm{L})]^{2+}$ and $[\mathrm{Co}-\mathrm{FPy}(\mathrm{L})]^{2+}$ of CoPy-COF and Co-FPy-COF, respectively-to calculate the energy levels of the COFs. We find that the electron affinity (EA) and the ionization potential (IP) of both COFs straddle the reduction potential of $\mathrm{CO}_{2}$ to $\mathrm{CO}$, as well as the proton reduction potential, and the oxidation potential of triethanolamine (Figure 3a). This provides a thermodynamic explanation for the ability of both $\mathrm{COFs}$ to drive $\mathrm{CO}_{2}$ reduction to $\mathrm{CO}$ in the presence of the sacrificial agent triethanolamine.

The photocatalytic $\mathrm{CO}_{2}$ reduction activity of Co-FPy-CON was then tested in water, acetonitrile, and triethanolamine (1:3:1 vol. mixtures) under visible light irradiation $(\lambda>$ $420 \mathrm{~nm}, 300 \mathrm{~W}$ Xe light source). Under these conditions the system was showed low activity and only trace amounts of carbon monoxide were produced (Figure S28).

We suspected that light harvesting and/or the following energy transfer might be responsible for the low activity of CoFPy-CON. We therefore employed $\left(\operatorname{Ir}\left[\mathrm{dF}\left(\mathrm{CF}_{3}\right) \mathrm{ppy}\right]_{2}(\mathrm{dtbpy})\right)_{\mathrm{PF}_{6}}$ as a photosensitizer in conjunction with Co-FPy-CON and found that the system worked efficiently producing carbon monoxide with a rate of $10.1 \mu \mathrm{mol}$ over 6 hours (TON $=28.1$ ) with a selectivity of $76 \%$ over the competing $\mathrm{H}_{2}$ generation reaction (Figures $4 \mathrm{a}, \mathrm{b}$ and Table S7). The ( $\operatorname{Ir}\left[\mathrm{dF}\left(\mathrm{CF}_{3}\right) \mathrm{ppy}\right]_{2}(\mathrm{dtbpy}) \mathrm{PF}_{6}$ dye on its own was found to be inactive producing no measurable $\mathrm{CO}$ after 7 hours (Table S7).

The predicted relative energy levels of the dye and the molecular COF models confirm that it is thermodynamically allowed for excited electrons and exicitons on the dye to be transferred to the COFs (Figure 3a), in line with the dyesensitization effects that we observed experimentally. Monte Carlo simulations of the dye adsorption on a single Co-FPyCOF layer, followed by non-covalent interaction analyses using DFT calculations of cluster models (Figure 3b,c), show that the dye binds strongly to both the pyrene moiety and the difluorobiphenyl moiety of Co-FPy-COF. Both binding sites are in the immediate vicinity of the photocatalytic Co site, beneficial to charge transfer from the dye to the COF.

Analysis based on DFT results of excited-state, interfragment charge transfer between the building units of CoFPy-COF and Co-Py-COF indicate that negligible amounts of electrons are transferred between the pyrene fragment and the Co-loaded iminopyridine fragment (Table S5), for the first three low-energy, excited electronic states with an appreciable oscillator strength. The corresponding intra-fragment electron redistributions within the individual fragments show that these low-energy states are local excitations, primarily located on the Co-loading iminopyridine moieties (Table S6). Further analyses of the natural transition orbitals for these electronic transitions indicate a metal-to-iminopyridine excitation mechanism, which is similar to the excitation of the molecular catalyst $\left[\mathrm{Co}(\mathrm{Bpy})_{3}\right]^{2+}$ in solution.

The fact that the COF does not act as a photocatalyst on its own requiring $\left[\left(\operatorname{Ir}\left[\mathrm{dF}\left(\mathrm{CF}_{3}\right) \mathrm{ppy}\right]_{2}(\mathrm{dtbpy})\right) \mathrm{PF}_{6}\right.$ as a photosensitizer to facilitate the catalytic reduction of $\mathrm{CO}_{2}$ together with 
the computational results indicate that the function of the COFs appears to be to allow for charge to be transferred and to provide metal coordination sites for the cobalt catalyst.

Isotope labeling study for ${ }^{13} \mathrm{CO}_{2}$ reduction using mass spectrometry confirmed that $\mathrm{CO}$ was produced from ${ }^{13} \mathrm{CO}_{2}$ (Figure 4c) ruling out degradation of the photosensitizer, photocatalyst or scavenger as the source of the produced CO. No hydrocarbon or alcohol products were observed. An external quantum efficiency (EQE) of $6.6 \%$ at $420 \mathrm{~nm}$ for the $\mathrm{CO}$ evolution of Co-FPy-CON was determined. This is higher than the previously reported EQE values of Ni-TpBpy-COF and Co-ZIF-9, both with $\left[\mathrm{Ru}(\mathrm{bpy})_{3}\right] \mathrm{Cl}_{2}$ as photosensitizer and triethanolamine as scavenger at $420 \mathrm{~nm}$. 3,14 The bulk sample of Co-FPyCOF produced $2.36 \mu$ mol CO over 6 hours (TON $=15.5$ ), 4.3times lower than that of Co-FPy-CON, which may be partly attributed to the lower loading of Co in Co-FPy-COF (0.9 wt. \% vs. 2.1 wt. \%, as in the COF and CON, respectively). Co-Py-CON was less active than its fluorinated counterpart, producing $7.4 \mu \mathrm{mol}$ of CO over 6 hours (TON $=10.9$ ). This might be attributed to the fact that Co-FPy-CON has a stronger binding affinity for $\mathrm{CO}_{2}$ (Figures $\mathrm{S} 11$ and 12) and to the dye (Figure S27), despite having a lower Co loading (2.1 wt. \%) than CoPy-CON (4.0 wt.\%). The bulk sample of Co-Py-COF also showed a lower $\mathrm{CO}$ production than its CON counterpart.

Bp-COF was synthesized as a comparison, which incorporates pyrene units and bipyridine sites instead of the iminopyridine sites, as in Py-COF and FPy-COF, for ligation of the Co complex (section 2, Supporting Information). Under the same conditions, Co-Bp-CON showed a comparable photocatalytic performance to Co-Py-CON, though producing less $\mathrm{CO}$ with a lower $\mathrm{CO} / \mathrm{H}_{2}$ selectivity. This suggests that it may be a promising, general strategy to incorporate iminopyridine based moieties into COFs as metal coordination sites for introducing catalytically-active metal centers into porous materials. Indeed, it was straightforward to prepare Zn-FPy-CON, which showed measurable $\mathrm{CO}_{2}$ reduction activities (Table S7).

Over a total of 10 hours irradiation under visible light (Figure 4 b), Co-FPy-CON showed a TON of 32.1; 2.8 times higher than its homogeneous counterpart $\left[\mathrm{Co}(\mathrm{bpy})_{n}\right]^{2+}$, which was deactivated after 3 hours, consistent with previous studies. ${ }^{37-39}$ Repeat experiments showed that the recycled CoFPy-CON catalyst retained its photocatalytic activity over four two-hour runs (Figure 4d) when fresh ( $\operatorname{Ir}\left[\mathrm{dF}\left(\mathrm{CF}_{3}\right) \mathrm{ppy}_{2}(\mathrm{dtbpy}) \mathrm{PF}_{6}\right.$ was added. It appears therefore that the decrease in activity observed for the extended 10hour run (Figure 4b) is due to dye degradation and Co-FPyCON appears to be stable under the photocatalysis conditions. This is further supported by post-illumination FT-IR spectra (Figure S21) of the sample after the repeat experiments. Furthermore, XPS measurements of the sample confirmed that the oxidation state of Co remained at +2 after the catalysis experiments (Figure S22). Only trace amounts of cobalt $(6.6 \mathrm{ppm})$ were found in the filtered solution after photocatalysis, measured by inductively coupled plasma-optical emission spectrometry, suggesting that Co-FPy-CON was not demetallized during the catalysis. No iridium was detected indicating that no photodeposition of iridium onto the CONs occurs nor that dye was absorbed onto the surface.

\section{CONCLUSIONS}

COF nanosheets loaded with single Co sites for photocatalytic $\mathrm{CO}_{2}$ reduction have been shown to be efficient in the presence of a dye and hole-scavenger. Co-FPy-CON achieved a high $\mathrm{CO}$ production of $10.1 \mu \mathrm{mol}$ and a $\mathrm{CO} / \mathrm{H}_{2}$ selectivity of $76 \%$, over 6 hours irradiation under visible light (TON $=28.1$ ). A high external quantum efficiency of $6.6 \%$ was determined under $420 \mathrm{~nm}$ irradiation. The performance of Co-FPy-CON is comparable to the state-of-the-art heterogeneous catalysts in the literature under comparable conditions (Table S8). The COF material in this case does not appear to act as a photocatalyst itself, but instead as a semiconductor support that transfers charge carriers that are generated in the dye to the cobalt centers, which are the active site. Nevertheless, COFs offer advantages, such as being tunable and can be exfoliated and we found across the systems studied here, the ultra-thin COF nanosheets were shown to consistently outperform their corresponding bulk materials in visible light driven $\mathrm{CO}_{2}$ reduction to CO. Finally, the iminopyridine moiety seems to be a promising alternative to bipyridine as metal coordination sites for ligation of catalytic metal centers into the backbone of porous networks, such as COFs, metal-organic frameworks and conjugated microporous polymers.

\section{ASSOCIATED CONTENT}

\section{Supporting Information.}

The Supporting Information is available free of charge on the ACS Publications website.

Synthetic procedures, FT-IR spectra, NMR, TGA, UV-Vis spectra, PXRD patterns, $\mathrm{CO}_{2}$ adsorption, SEM images, STEM images, AFM images, PL spectra and TCSPC experiments, PL quenching data, post-photocatalysis characterization data, and computational details.

\section{AUTHOR INFORMATION}

\section{Corresponding Author}

*E-mail: lchen@liverpool.ac.uk (L.C.).

*E-mail: Sebastian.sprick@strath.ac.uk (R.S.S.).

*E-mail: aicooper@liverpool.ac.uk (A.I.C)

\section{Author Contributions}

The manuscript was written through contributions of all authors.

Notes

The authors declare no competing financial interest.

\section{ACKNOWLEDGMENTS}

The authors acknowledge funding from the Engineering and Physical Sciences Research Council (EPSRC) (EP/N004884/1), and the Leverhulme Trust via the Leverhulme Research Centre for Functional Materials Design. X.W., Z.F., C.Z. and L.L. thank the China Scholarship Council for a PhD studentship. We thank A. Ciupa for help with GC-MS, and Y. Yan and J. Ward for useful discussions.

\section{REFERENCES}

(1) Inoue, T.; Fujishima, A.; Konishi, S.; Honda, K. Photoelectrocatalytic Reduction of Carbon Dioxide in Aqueous Suspensions of Semiconductor. Nature 1979, 277, 637-638.

(2) White, J. L.; Baruch, M. F.; Pander, J. E.; Hu, Y.; Fortmeyer, I. C.; 
Park, J. E.; Zhang, T.; Liao, K.; Gu, J.; Yan, Y.; Shaw, T. W.; Abelev, E.; Bocarsly, A. B. Light-Driven Heterogeneous Reduction of Carbon Dioxide: Photocatalysts and Photoelectrodes. Chem. Rev. 2015, $115,12888-12935$.

(3) Wang, S.; Yao, W.; Lin, J.; Ding, Z.; Wang, X. Cobalt Imidazolate Metal-Organic Frameworks Photosplit $\mathrm{CO}_{2}$ under Mild Reaction Conditions. Angew. Chem. Int. Ed. 2014, 53, 1034-1038.

(4) Rao, H.; Schmidt, L. C.; Bonin, J.; Robert, M. Visible-LightDriven Methane Formation from $\mathrm{CO}_{2}$ with a Molecular Iron Catalyst. Nature 2017, 548, 74-77.

(5) Takeda, H.; Ohashi, K.; Sekine, A.; Ishitani, O. Photocatalytic $\mathrm{CO}_{2}$ Reduction Using $\mathrm{Cu}(\mathrm{I})$ Photosensitizers with a Fe(II) Catalyst. J. Am. Chem. Soc. 2016, 138, 4354-4357.

(6) Wang, Y.; Liu, X.; Han, X.;Godin, R.; Chen, J.; Zhou, W.; Jiang, C.; Thompson, J.; Mustafa, K.; Shevlin, S.; Durrant, J.; Guo Z.; Tang. J. Unique hole-accepting carbon-dots promoting selective carbon dioxide reduction nearly $100 \%$ to methanol by pure water. Nat. Commun. 2020, 11, 2531

(7) Kuehnel, M. F.; Sahm, C. D.; Neri, G.; Lee, J. R.; Orchard, K. L.; Cowan, A. J.; Reisner, E. ZnSe Quantum Dots Modified with a $\mathrm{Ni}\left(\right.$ Cyclam) Catalyst for Efficient Visible-Light Driven $\mathrm{CO}_{2}$ Reduction in Water. Chem. Sci. 2018, 9, 2501-2509.

(8) Roy, S.; Reisner, E. Visible-Light-Driven $\mathrm{CO}_{2}$ Reduction by Mesoporous Carbon Nitride Modified with Polymeric Cobalt Phthalocyanine. Angew. Chem. Int. Ed. 2019, 58, 12180-12184.

(9) McMorn, P.; Hutchings, G. J. Heterogeneous Enantioselective Catalysts: Strategies for the Immobilisation of Homogeneous Catalysts. Chem. Soc. Rev. 2004, 33, 108-122.

(10) Wang, W.; Wang, S.; Ma, X.; Gong, J. Recent Advances in Catalytic Hydrogenation of Carbon Dioxide. Chem. Soc. Rev. 2011, 40, 3703-3727.

(11) Deng X.; Qin, Y.; Hao, M.; Li, Z. MOF-253-Supported Ru Complex for Photocatalytic $\mathrm{CO}_{2}$ Reduction by Coupling with Semidehydrogenation of 1,2,3,4-Tetrahydroisoquinoline (THIQ) Inorg. Chem. 2019, 58, 16574-16580.

(12) Deng, X.; Albero, J.; Xu, L.; García, H.; Li, Z. Construction of a Stable Ru-Re Hybrid System Based on Multifunctional MOF-253 for Efficient Photocatalytic $\mathrm{CO}_{2}$ Reduction. Inorg. Chem. 2018, 57, 8276-8286.

(13) Sun, D.; Gao, Y.; Fu, J.; Zeng, X.; Chen, Z.; Li Z. Construction of a Supported $\mathrm{Ru}$ Complex on Bifunctional MOF-253 for Photocatalytic $\mathrm{CO}_{2}$ Reduction under Visible Light. Chem. Commun. 2015, 51, 2645-2648.

(14) Zhong, W.; Sa, R.; Li, L.; He, Y.; Li, L.; Bi, J.; Zhuang, Z.; Yu, Y.; Zou, Z. A Covalent Organic Framework Bearing Single Ni Sites as a Synergistic Photocatalyst for Selective Photoreduction of $\mathrm{CO}_{2}$ to CO. J. Am. Chem. Soc. 2019, 141, 7615-7621.

(15) Yang, S.; Hu, W.; Zhang, X.; He, P.; Pattengale, B.; Liu, C.; Cendejas, M.; Hermans, I.; Zhang, X.; Zhang, J.; Huang, J. 2D Covalent Organic Frameworks as Intrinsic Photocatalysts for Visible Light-Driven $\mathrm{CO}_{2}$ Reduction. J. Am. Chem. Soc. 2018, 140, 14614-14618.

(16) Lu, M.; Liu, J.; Li, Q.; Zhang, M.; Liu, M.; Wang, J.-L.; Yuan, D.Q.; Lan, Y.-Q. Rational Design of Crystalline Covalent Organic Frameworks for Efficient $\mathrm{CO}_{2}$ Photoreduction with $\mathrm{H}_{2} \mathrm{O}$. Angew. Chem. Int. Ed. 2019, 58, 12392-12397.

(17) Banerjee, T.; Haase, F.; Savasci, G.; Gottschling, K.; Ochsenfeld, C.; Lotsch, B. V. Single Site Photocatalytic $\mathrm{H}_{2}$ Evolution from Covalent Organic Frameworks with Molecular Cobaloxime Co-Catalysts. J. Am. Chem. Soc. 2017, 139, 16228-16234.

(18) Biswal, B. P.; Vignolo-González, H. A.; Banerjee, T.; Grunenberg, L.; Savasci, G.; Gottschling, K.; Nuss, J.; Ochsenfeld, C.; Lotsch, B. V. Sustained Solar $\mathrm{H}_{2}$ Evolution from a Thiazolo[5,4d]Thiazole-Bridged Covalent Organic Framework and NickelThiolate Cluster in Water. J. Am. Chem. Soc. 2019, 141, 1108211092.

(19) Zhang, H.; Wei, J.; Dong, J.; Liu, G.; Shi, L.; An, P.; Zhao, G.;
Kong, J.; Wang, X.; Meng, X.; Zhang, J.; Ye, J. Efficient Visible-LightDriven Carbon Dioxide Reduction by a Single-Atom Implanted Metal-Organic Framework. Angew. Chem. Int. Ed. 2016, 55, 14310-14314.

(20) Jiao, L.; Jiang, H. L. Metal-Organic-Framework-Based SingleAtom Catalysts for Energy Applications. Chem 2019, 5, 786-804.

(21) Fu, Y.; Sun, D.; Chen, Y.; Huang, R.; Ding, Z.; Fu, X.; Li, Z. An Amine-Functionalized Titanium Metal-Organic Framework Photocatalyst with Visible-Light-Induced Activity for $\mathrm{CO}_{2}$ Reduction. Angew. Chem. Int. Ed. 2012, 51, 3364-3367.

(22) Sun, D.; Fu, Y.; Liu, W.; Ye, L.; Wang, D.; Yang, L.; Fu, X.; Li, Z. Studies on Photocatalytic $\mathrm{CO}_{2}$ Reduction over $\mathrm{NH}_{2}$-Uio-66(Zr) and Its Derivatives: Towards a Better Understanding of Photocatalysis on Metal-Organic Frameworks. Chem. Eur. J. 2013, 19, 1427914285

(23) Wang, D.; Huang, R.; Liu, W.; Sun, D.; Li, Z. Fe-Based MOFs for Photocatalytic $\mathrm{CO}_{2}$ Reduction: Role of Coordination Unsaturated Sites and Dual Excitation Pathways. ACS Catal. 2014, 4, 42544260.

(24) Chen, Y.; Wang, D.; Deng X.; Li Z. Metal-organic frameworks (MOFs) for photocatalytic $\mathrm{CO}_{2}$ reduction. Catal. Sci. Technol. 2017, 7, 4893-4904.

(25) Wang, X.; Chen, L.; Chong, S. Y.; Little, M. A.; Wu, Y.; Zhu, W. H.; Clowes, R.; Yan, Y.; Zwijnenburg, M. A.; Sprick, R. S.; Cooper, A. I. Sulfone-Containing Covalent Organic Frameworks for Photocatalytic Hydrogen Evolution from Water. Nat. Chem. 2018, 10, 1180-1189.

(26) Banerjee, T.; Gottschling, K.; Savasci, G.; Ochsenfeld, C.; Lotsch, B. V. H 2 Evolution with Covalent Organic Framework Photocatalysts. ACS Energy Lett. 2018, 3, 400-409.

(27) Pachfule, P.; Acharjya, A.; Roeser, J.; Langenhahn, T. Schwarze, M.; Schomaecker, R.; Thomas, A.; Schmidt, J. Diacetylene Functionalized Covalent Organic Framework (COF) for Photocatalytic Hydrogen Generation. J. Am. Chem. Soc. 2017, 140, 1423-1427.

(28) Fu, Z.; Wang, X.; Gardner, A.; Wang, X.; Chong, S. Y.; Neri, G.; Cowan, A. J.; Liu, L.; Li, X.; Vogel, A.; Clowes, R.; Bilton, M.; Chen, L.; Sprick, R. S.; Cooper, A. A Stable Covalent Organic Framework for Photocatalytic Carbon Dioxide Reduction. Chem. Sci. 2020, 11, 543-550.

(29) Wang, S.; Hai, X.; Ding, X.; Jin, S.; Xiang, Y.; Wang, P.; Jiang, B.; Ichihara, F.; Oshikiri, M.; Meng, X.; Li, Y.; Matsuda, W.; Ma, J.; Seki, S.; Wang, X.; Huang, H.; Wada, Y.; Chen, H.; Ye, J. Intermolecular cascaded $\pi$-conjugation channels for Electron Delivery Powering $\mathrm{CO}_{2}$ Photoreduction. Nat. Commun. 2020, 11, 1149.

(30) Lu, M.; Li, Q.; Liu, J.; Zhang, F. M.; Zhang, L.; Wang, J. L.; Kang, Z. H.; Lan, Y. Q. Installing Earth-Abundant Metal Active Centers to Covalent Organic Frameworks for Efficient Heterogeneous Photocatalytic $\mathrm{CO}_{2}$ Reduction. Appl. Catal. B Environ. 2019, 254, 624-633.

(31) Bunck, D. N.; Dichtel, W. R. Bulk Synthesis of Exfoliated TwoDimensional Polymers Using Hydrazone-Linked Covalent Organic Frameworks. J. Am. Chem. Soc. 2013, 135, 14952-14955.

(32) Alahmed, A. H.; Briggs, M. E.; Cooper, A. I.; Adams, D. J. PostSynthetic Fluorination of Scholl-Coupled Microporous Polymers for Increased $\mathrm{CO}_{2}$ Uptake and Selectivity. J. Mater. Chem. A 2019, 7, 549-557.

(33) Li, G.; Zhang, B.; Wang, Z. Facile Synthesis of Fluorinated Microporous Polyaminals for Adsorption of Carbon Dioxide and Selectivities over Nitrogen and Methane. Macromolecules 2016, $49,2575-2581$.

(34) Chen, C.; Wu, T.; Wu, H.; Liu, H.; Qian, Q.; Liu, Z.; Yang, G.; Han, B. Highly Effective Photoreduction of $\mathrm{CO}_{2}$ to $\mathrm{CO}$ Promoted by Integration of CdS with Molecular Redox Catalysts through MetalOrganic Frameworks. Chem. Sci. 2018, 9, 8890-8894.

(35) Hojilla Atienza, C. C.; Bowman, A. C.; Lobkovsky, E.; Chirik, P. J. Photolysis and Thermolysis of Bis(Imino)Pyridine Cobalt 
Azides: C-H Activation from Putative Cobalt Nitrido Complexes. J. Am. Chem. Soc. 2010, 132, 16343-16345.

(36) Bowman, A. C.; Milsmann, C.; Atienza, C. C. H.; Lobkovsky, E.; Wieghardt, K.; Chirik, P. J. Synthesis and Molecular and Electronic Structures of Reduced Bis(Imino)Pyridine Cobalt Dinitrogen Complexes: Ligand versus Metal Reduction. J. Am. Chem. Soc. 2010, 132, 1676-168.

(37) Liu, C.; Dubois, K. D.; Louis, M. E.; Vorushilov, A. S.; Li, G. Photocatalytic $\mathrm{CO}_{2}$ Reduction and Surface Immobilization of a Tricarbonyl $\operatorname{Re}(\mathrm{I})$ Compound Modified with Amide Groups. ACS Catal. 2013, 3, 655-662.

(38) Guo, Z.; Cheng, S.; Cometto, C.; Anxolabéhère-Mallart, E.; Ng, S. M.; Ko, C. C.; Liu, G.; Chen, L.; Robert, M.; Lau, T. C. Highly Efficient and Selective Photocatalytic $\mathrm{CO}_{2}$ Reduction by Iron and Cobalt Quaterpyridine Complexes. J. Am. Chem. Soc. 2016, 138, 9413-9416.

(39) Won, D. Il; Lee, J. S.; Ji, J. M.; Jung, W. J.; Son, H. J.; Pac, C.; Kang, S. O. Highly Robust Hybrid Photocatalyst for Carbon Dioxide Reduction: Tuning and Optimization of Catalytic Activities of Dye $/ \mathrm{TiO}_{2} / \mathrm{Re}(\mathrm{I})$ Organic-Inorganic Ternary Systems. J. Am. Chem. Soc. 2015, 137, 13679-13690. 


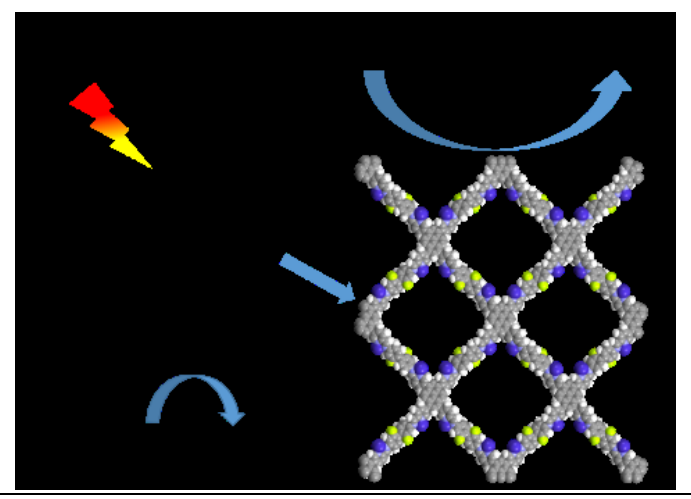

